\title{
Weight gain prevention in the school worksite setting: Results of a multi-level cluster randomized trial ${ }^{\text {t }}$
}

\author{
Stephenie C. Lemon ${ }^{\text {a,* }}$, Monica L. Wang ${ }^{\text {a }}$, Nicole M. Wedick ${ }^{\text {a }}$, Barbara Estabrook ${ }^{\text {a }}$, Susan Druker ${ }^{\text {a }}$, \\ Kristin L. Schneider ${ }^{\mathrm{b}}$, Wenjun $\mathrm{Li}^{\mathrm{a}}$, Lori Pbert ${ }^{\mathrm{a}}$ \\ a University of Massachusetts Medical School, Division of Preventive and Behavioral Medicine, Department of Medicine, 55 Lake Avenue North, Worcester, MA 01655, USA \\ ${ }^{\mathrm{b}}$ Rosalind Franklin University of Medicine and Science, Department of Psychology, 3333 Green Bay Road, North Chicago, IL 60064, USA
}

\section{A R T I C L E I N F O}

Available online 15 December 2013

\section{Keywords:}

Obesity prevention

Worksite health promotion

Environmental interventions

Ecological model

Program evaluation

\begin{abstract}
A B S T R A C T
Objective. To describe the effectiveness, reach and implementation of a weight gain prevention intervention among public school employees.

Method. A multi-level intervention was tested in a cluster randomized trial among 782 employees in 12 central Massachusetts public high schools from 2009 to 2012. The intervention targeted the nutrition and physical activity environment and policies, the social environment and individual knowledge, attitudes and skills. The intervention was compared to a materials only condition. The primary outcome measures were change in weight and body mass index (BMI) at 24-month follow-up. Implementation of physical environment, policy and social environment strategies at the school and interpersonal levels, and intervention participation at the individual level were assessed.

Results. At 24-month follow-up, there was a net change (difference of the difference) of -3.03 pounds ( $p=.04)$ and of -.48 BMI units $(p=.05)$ between intervention and comparison conditions. The majority of intervention strategies were successfully implemented by all intervention schools, although establishing formal policies was challenging. Employee participation in programs targeting the physical and social environment was maintained over time.

Conclusion. This study supports that a multi-level intervention integrated within the organizational culture can be successfully implemented and prevent weight gain in public high school employees.
\end{abstract}

(C) 2013 Published by Elsevier Inc.

\section{Introduction}

The integration of health promotion and disease prevention efforts in worksite settings represents an important opportunity to improve population health (Centers for Disease Control and Prevention 2012; Task Force on Community Prevention Services, 2009; U.S. Department of Health and Human Services, 2012). A key component of the U.S. Patient Protection and Affordable Care Act (ACA) of 2010 is the development and support of opportunities for worksite health promotion as part of a comprehensive national strategy to transform health care

\footnotetext{
This is an open-access article distributed under the terms of the Creative Commons Attribution-NonCommercial-No Derivative Works License, which permits non-commercial use, distribution, and reproduction in any medium, provided the original author and source are credited.

* Corresponding author at: Division of Preventive and Behavioral Medicine, Department of Medicine, University of Massachusetts Medical School, 55 Lake Avenue North, Worcester, MA 01655, USA. Fax: +1 5088563840.

E-mail addresses: Stephenie.Lemon@umassmed.edu (S.C. Lemon), Monica.Wang@umassmed.edu (M.L. Wang), Nicole.Wedick@umassmed.edu (N.M. Wedick), Barbara.Estabrook@umassmed.edu (B. Estabrook), Susan.Druker@umassmed.edu (S. Druker), Kristin.Schneider@rosalindfranklin.edu (K.L. Schneider), Wenjun.Li@umassmed.edu (W. Li), Lori.Pbert@umassmed.edu (L. Pbert).
}

delivery and improve the nation's health (The Robert Wood Johnson Foundation, 2012; U.S. Government Printing Office, March, 2010). As a result of the ACA, the number of worksite health promotion initiatives that are offered and the number of employees who have access to these initiatives are expected to greatly increase.

Primary targets for worksite health initiatives include obesity and weight gain prevention. Obesity rates among adults continue to remain high, with an estimated 35.7\% of U.S. adults in 2009-2010 (Ogden et al., 2012) who were obese. Most U.S. adults gain 1 to 2 pounds per year (Lewis et al., 2000), the accumulation of which has contributed significantly to the rise in obesity over recent decades (Flegal et al., 2012). Preventing this gradual increase in weight through small changes to reduce energy intake could have a tremendous public health impact (Hill et al., 2003). The negative health and economic consequences related to obesity and obesity-related outcomes places enormous burden both on individuals and worksites. Medical costs related to obesity reached approximately $\$ 147$ billion in 2008 (Finkelstein et al., 2009), of which employers were accountable for a substantial proportion as major payers of health insurance benefits to employees (Blumenthal, 2006). Obese employed adults additionally incur costs to worksites related to increased absenteeism and lost job productivity (Thorndike, 2011). 
Programs to reduce obesity delivered through the worksite setting thus represent a critical opportunity to improve the health and productivity and reduce costs incurred by employers.

Worksites are particularly well suited for the implementation of weight gain prevention programs and initiatives that target the physical and social environment. Worksites provide access to a large concentration of relatively stable populations who share geographic proximity, often have common characteristics and goals (Goetzel and Ozminkowski, 2008), and have access to common communication systems, onsite facilities and other resources. The existence of ongoing work relationships holds potential for maximizing social support for behavior change, an important factor influencing obesity prevention efforts (Kamphuis et al., 2006; J. Sallis and Owen, 1999; Shaikh et al., 2008; Trost et al., 2002) and the contained worksite environment may alleviate common barriers to weight gain prevention for employees, such as cost, limited time and inconvenience (Cahill et al., 2008). However, the evidence supporting population-based weight gain prevention strategies in worksites to date has been equivocal (Gudzune et al., 2013; Hennrikus and Jeffery, 1996; Janer et al., 2002; Lemon and Estabrook, 2013; Mattke et al., 2013; Verweij et al., 2010), with a recent systematic review reporting that the substantial heterogeneity in intervention strategies and study populations examined to date make it challenging to draw conclusions about which approaches are most effective (Gudzune et al., 2013).

To maximize the impact of the ACA's investments in worksite health promotion, a stronger evidence base for weight gain prevention strategies is needed. Given the heterogeneity of worksites, interventions must be targeted to the unique culture of each type of worksite setting, while also broad enough to maximize generalizability and dissemination. U.S. elementary and secondary schools employ $5.1 \%$ of the nation's workforce (U.S. Department of Labor and Division of Labor Force Statistics, 2012) and share similar organizational and physical infrastructure characteristics nationwide. While schools have been commonly utilized as a setting to implement and evaluate childhood obesity intervention efforts, school health policies and programs for comprehensive obesity control for school staff are lacking. Few studies have targeted the school setting to address employee health, and results from published obesityrelated interventions targeting school staff have utilized diverse intervention strategies that have not targeted the physical and social environment (Aldana et al., 2005; Blair et al., 1986; Dunn et al., 2013; Resnicow et al., 1998; Siegel et al., 2010).

The present study aims were to compare the effectiveness of a multilevel weight gain prevention intervention that targeted the physical and social environment to a materials-only comparison condition among public high school employees, to examine potential intervention modification by employee characteristics, and to describe the implementation of and participation in the multi-level intervention. The primary study hypothesis was that employees at schools that received a multilevel intervention would not gain weight over the two-year intervention period, while employees at schools that received a materials-only intervention would gain weight at an average rate of 1 to 2 pounds per year (Lewis et al., 2000).

\section{Methods}

\section{Study design}

The study utilized a cluster randomized design and was conducted among 12 public high schools within 50 miles of Worcester, Massachusetts. The unit of randomization was the school with individual employees as the unit of analysis. The intervention was conducted between 2010 and 2012. The study protocol was approved by the Institutional Review Board of the University of Massachusetts Medical School and registered on clinicaltrials.gov (Identifier: NCT01467284).

\section{Study site recruitment and randomization procedures}

Superintendents, principals and school nurses of all public high schools within 50 miles of Worcester, MA $(N=114)$ were sent a letter describing the goals of the study and asked to contact study personnel if they were interested in participating. Schools that responded $(N=32,28.1 \%)$ were stratified into 3 groups based on school size and urbanicity (large urban, small urban, rural). Within each of these strata, 4 schools were randomly selected to either the multi-level intervention condition or the materials-only intervention condition, for a total of 12 participating schools, 6 per condition.

\section{Study cohort recruitment and follow-up procedures}

Baseline assessments occurred in Spring, 2010 and follow-up assessments occurred at 12 and 24 months. Study enrollment was independent of the intervention, as the intervention was targeted to all employees. Enrollment and baseline assessment were completed prior to randomization of the sites to the multi-level or materials-only condition. All employees were invited by a letter delivered to school email addresses or work mailboxes, signed by the school principal and the two study principal investigators. Interested individuals were instructed to attend a scheduled drop-in session held before and after the school work day and during break periods. These sessions were announced by email and daily reminders were made by email and verbally by school staff members involved in the study at meetings and in faculty lounges. At each school, a quota for the number of employees to enroll (approximately half of all employees) was established based on the total size of the workforce. Once the quota was achieved, recruitment at that school was completed. Assessments occurred over a one-to-two-week period, depending upon the size of the workforce. Trained study personnel obtained written informed consent and screened potential participants using the following inclusion criteria: 1) able to understand and communicate in English, 2) no plan to leave employment in the next 2 years, 3) worked at least $15 \mathrm{~h}$ per week, 4) not pregnant or had not given birth in the past 6 months, and 5) no physical impediment to being weighed and measured. Eligible persons completed the baseline assessment. Recruitment occurred from January to May 2010. At follow-up assessment points, cohort members were contacted by the research coordinator via email. If a participant did not respond to the email, a phone call or in-person contact was made. No efforts were made to contact those no longer working at the schools at follow-up.

\section{Multi-level intervention condition}

Guided by the social ecological model (Lemon et al., 2010; Sallis et al., 2008; Zapka et al., 2007) the intervention targeted three levels of influence: individual, interpersonal, and organizational (school). The intervention was coordinated and championed onsite by "coaches", who were school employees (predominantly school nurses) that were paid a $\$ 2000$ stipend for their work on the project. An Employee Advisory Group, comprised of staff members solicited by the coach, met on an approximately quarterly basis to provide overall direction to the intervention, with particular focus on policy and environmental intervention components and promoting intervention activities among their co-workers.

Although the overall scope of the intervention was consistent across schools, the coaches and Employee Advisory Groups implemented activities that were tailored to each school, with input from site-specific focus groups of employees conducted at the beginning of the intervention period. At the organizational level, physical environment and policy interventions included access to onsite fitness facilities and locker rooms, availability of healthy lunch options to teachers and staff, elimination and reduction of sugar-sweetened beverages in faculty lounges and point-of-purchase nutritional information in cafeterias. At the interpersonal level, social environment interventions that were implemented included group walking and physical activity campaigns and challenges, onsite group fitness classes, walking groups, staff basketball games, and organized healthy potluck lunches and breakfasts. Strategies targeting individuals included periodic health promotion displays and healthy food tastings, weight loss and weight maintenance challenges and self-weighing programs, in addition to print and web-based materials, which were also provided in the materials only-comparison condition, described below.

\section{Comparison condition}

The comparison condition consisted of print and electronic materials only. These included an employee resource book that addressed topics related to healthy eating, physical activity, and weight management. The initial book distributed at the beginning of the intervention was supplemented by six installments, three in each of the two school years. Example materials included 
healthy recipes, walking maps of routes in the school building and outdoors on school grounds and nearby streets, and educational materials on healthy eating, weight management and physical activity topics. Electronic materials consisted of a project website that displayed all print materials, and a one-page weekly newsletter delivered via email. Each newsletter featured a different spotlight article on a topic relevant to healthy eating, physical activity or weight management; a healthy recipe and a tip to improve weight-related behaviors.

\section{Assessments and data sources}

Employee cohort members completed assessments at three time intervals (baseline, 12 month follow-up, and 24 month follow-up). Assessments occurred in school meeting rooms or offices before or after work, or during scheduled breaks. Data sources and collection methods included anthropometric measurements administered by trained personnel and a 30-min selfadministered survey. Employees received a $\$ 20$ gift card for completing measurements at each time point.

Measures

The primary outcome measures were change in weight and body mass index (BMI). Weight measurements were taken by trained staff using portable digital scales with readings to the nearest $2 / 10^{\text {th }}$ pound. Heights were measured to the nearest $1 / 8^{\text {th }}$ inch using portable stadiometers. Weight and height were converted to the metric scale and BMI calculated as weight in kilograms divided by height in meters squared $\left(\mathrm{kg} / \mathrm{m}^{2}\right)$.

Demographic characteristics included gender, age group, education level, occupation (teacher or staff) and race/ethnicity. Participants' BMI at baseline was categorized as underweight (less than $18.5 \mathrm{~kg} / \mathrm{m}^{2}$ ), healthy weight (between 18.5 and $24.9 \mathrm{~kg} / \mathrm{m}^{2}$ ), overweight (between 25.0 and $29.9 \mathrm{~kg} / \mathrm{m}^{2}$ ), or obese (at or above $30.0 \mathrm{~kg} / \mathrm{m}^{2}$ ). Participants self-reported whether or not they were currently trying to lose weight at baseline as a yes/no binary indicator.

Implementation of physical environment and policy intervention strategies at the site-level was assessed qualitatively through staff logs and meeting minutes at intervention sites. Intervention participation at the individual level included self-report of participation in specific intervention strategies.

Statistical analyses

The trial was designed with $80 \%$ power to detect a minimum difference of .5 BMI units between employees in the intervention sites and those in the comparison sites at a two-sided alpha level of .05, accounting for within-school clustering of individuals. Intention-to-treat analyses were conducted using the baseline sample of 782 employees. Means and frequency distributions were computed to describe the study sample at baseline and to describe implementation of and employee participation in specific intervention strategies. Hierarchical survey regression models were used to estimate the effect of the intervention on employee weight and BMI at 12- and 24-month follow-up visits while accounting for nesting of individual within school. Inverse probability weighting was used to account for participant probability of being lost to follow-up. The intervention effect was estimated for the overall study sample and subgroups. Intervention effects across subgroups were also compared with statistical interaction terms. To account for loss to follow-up, models were weighted to adjust for the probability of participants being retained at the 12- and 24-month follow-up visits. Models were adjusted for participant age (grand mean centered), gender, race/ethnicity, and occupation. All analyses were conducted using Stata version 12.1 (StataCorp LP, College Station, TX) and SAS version 9.3 (SAS Institute, Cary, NC). Statistical significance was determined by an alpha level of 0.05 .

\section{Results}

\section{Enrollment and retention rates}

Employee enrollment quotas were achieved at each site, with over half of all employees enrolled in the cohort ( $52.3 \%, n=482$ at intervention sites; $54.9 \%, n=359$ at comparison sites). Individuals who became ineligible after baseline because of pregnancy or health related conditions known to affect weight or who did not have at least one followup assessment were excluded for analyses presented, resulting in an analytic sample of 782 . At the 24 month follow-up, intervention sites had $73.4 \%$ retention and comparison sites had $80.0 \%$ retention. Among employees who remained eligible for follow-up assessments, retention rates were $99.2 \%$ for intervention sites and $99.0 \%$ for comparison sites at 24 month follow-up. See CONSORT diagram in Fig. 1 (Schulz et al., 2010).

\section{Study sample}

Table 1 describes the study sample. The intervention and comparison conditions were comparable on all participant characteristics except for gender, with intervention sites having a greater percentage of female participants (70.2\%) than comparison sites (62.8\%). Among the total sample, approximately half of the sample (49.6\%) were aged 45 and over and employed as teachers (54.7\%), and the majority (95.9\%) were non-Latino White. With respect to baseline BMI, 35.8\% were classified as overweight and $29.0 \%$ were classified as obese.

\section{Intervention effectiveness}

Fig. 2 depicts the estimates of mean weight change in both study conditions on the linear regression models using intention to treat principles and adjusting for dropout probability. Average baseline weight was identical (173.9 lbs.) in both conditions. Among intervention condition participants, average weight decreased to $173.2 \mathrm{lbs}$. at 12 months and $172.6 \mathrm{lbs}$. at 24 months. Among comparison condition participants, average weight increased to $175.9 \mathrm{lbs}$. at 12 months and $176.1 \mathrm{lbs}$. at 24 months. Table 2 describes the differences in the estimates of average weight and BMI change across conditions at 12 and 24 months. For both outcome variables, there were statistically significant differences at 24month follow-up. There was a net change (difference of the difference) of $-3.03 \mathrm{lbs}$. ( $p=.04)$ and of -.48 BMI units $(p=.05)$ between intervention and comparison conditions.

With respect to effect modification, a statistically significant difference in weight change was observed between conditions for participants aged 35 to 44 at 24 -month follow-up ( -5.4 lbs., $p=.05$ ), but not among participants aged 21 to $34(-1.8$ pounds, $p=.59)$ or participants aged 45 and over $(-2.5$ pounds, $p=.18$ ) ( $p$ for interaction $=.04)$. Statistical interaction terms for each of the other employee characteristics, including demographic factors, baseline BMI and current weight loss attempt, were not significant. No unintended consequences or harms were identified in either condition.

\section{Intervention implementation and participation}

Table 3 describes site-level implementation of the intervention strategies and participant use of these strategies. A majority of the intervention strategies were successfully implemented by all schools in the intervention condition. Employees reported their participation in the various components of the intervention on the 12 month and 24 month follow-up questionnaires, as described in Table 3. Generally, participation in programs that targeted the physical and social environment was similar in year one and year two. Programs promoting physical activity had higher participation in year one than year two. Programs targeting healthy eating (healthy potlucks and healthy food tastings/displays) saw stable or increased participation over time. Use of informational resources aimed at the individual increased from year 1 to year 2 .

\section{Discussion}

Elementary and high school worksites provide an excellent venue for adult weight gain prevention interventions. Importantly, school employees represent a wide range of occupational and socioeconomic backgrounds, including principals, teachers, counselors, school nurses, administrative staff, food service workers, and maintenance staff. Nationally, school health is a major public health priority with respect to 


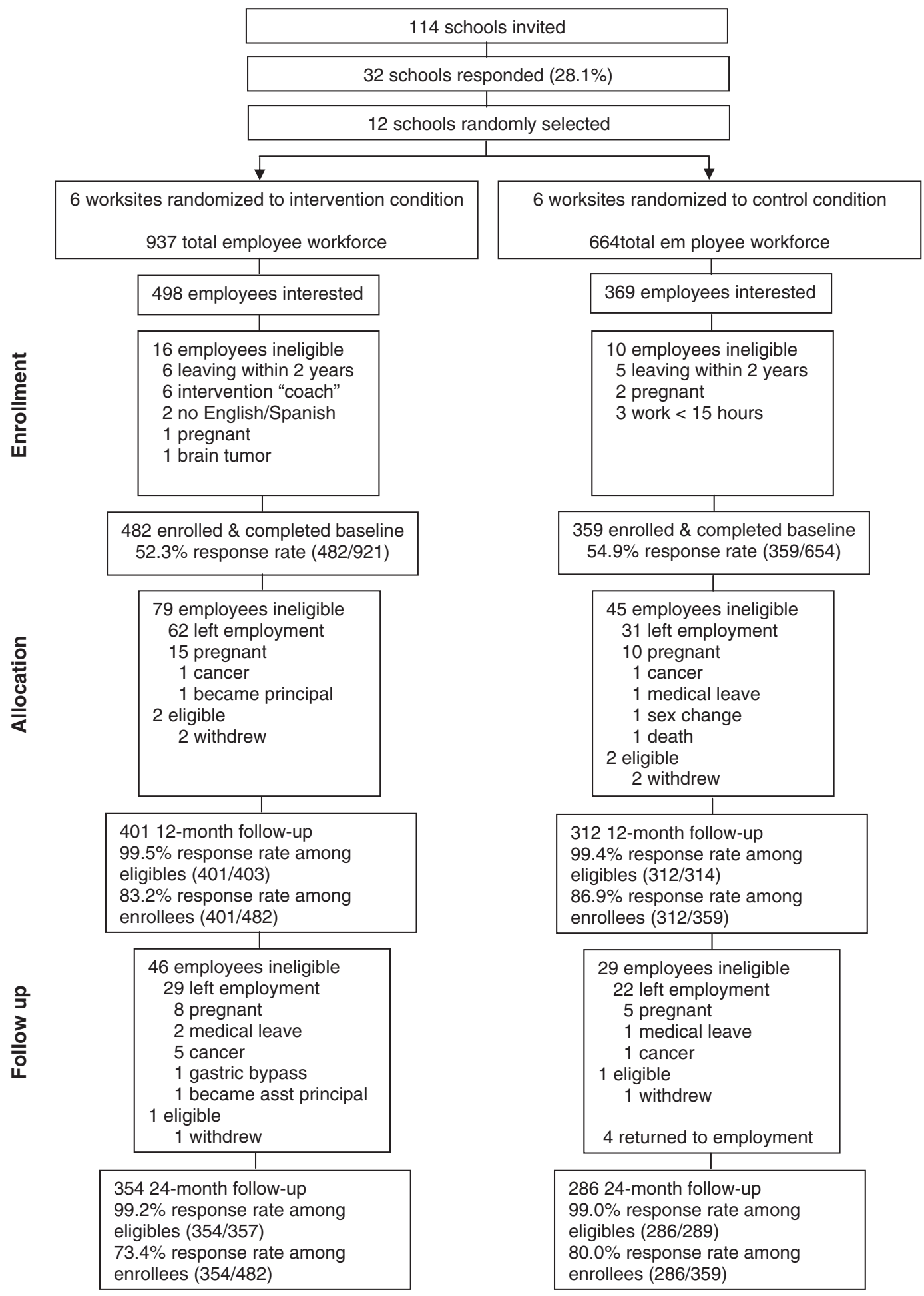

Fig. 1. CONSORT diagram of the multi-level weight gain prevention intervention trial conducted among public school employees in central Massachusetts, $2010-2012$.

addressing youth diet, physical activity and obesity, and this is reflected in federal requirements that all schools participating in the National School Lunch Program must implement, evaluate and report on a local school wellness policy (U.S. Government, 2010). Although teacher and staff health is included as one of the eight components of Coordinated School Health (Centers for Disease Control and Prevention 2013), these initiatives have not prioritized school teachers and staff to date. As employees are potential models of healthy lifestyles for students, future intervention approaches could simultaneously target employees and students.
This school-randomized study demonstrated that a multi-level weight gain prevention intervention was associated with modest differences in weight change between intervention and comparison participants. Comparison condition participants gained an average of 1.5 pounds over the two-year follow-up period, slightly less than the national average. In contrast, intervention participants lost an average of 1.5 pounds, which we hypothesized would remain steady (see Fig. 2). While the difference in weight change between conditions is not clinically significant such as those obtained in intensive weight loss programs, small shifts in population weight gain (or lack thereof) 
Table 1

Baseline description of the study sample $(n=782)$ in a multi-level weight gain prevention intervention trial conducted among public school employees in central Massachusetts, 2010-2012.

\begin{tabular}{|c|c|c|c|c|}
\hline Characteristic & $\begin{array}{l}\text { Total } \\
(n=782)\end{array}$ & $\begin{array}{l}\text { Intervention Sites } \\
(n=446)\end{array}$ & $\begin{array}{l}\text { Comparison Sites } \\
(n=336)\end{array}$ & $P$-value \\
\hline Gender & & & & .03 \\
\hline Female & $524(67.0 \%)$ & $313(70.2 \%)$ & $211(62.8 \%)$ & \\
\hline Male & $258(33.0 \%)$ & $133(29.8 \%)$ & $125(37.2 \%)$ & \\
\hline Age & & & & .12 \\
\hline $21-34$ & $190(24.3 \%)$ & $120(26.9 \%)$ & $70(20.8 \%)$ & \\
\hline $35-44$ & $204(26.1 \%)$ & $116(26.0 \%)$ & $88(26.2 \%)$ & \\
\hline $45+$ & $388(49.6 \%)$ & $210(47.1 \%)$ & $178(53.0 \%)$ & \\
\hline Race/ethnicity & & & & .17 \\
\hline White & 750 (95.9\%) & $424(95.1 \%)$ & $326(97.0 \%)$ & \\
\hline Non-White & $32(4.1 \%)$ & $22(4.9 \%)$ & $10(3.0 \%)$ & \\
\hline Occupation & & & & .11 \\
\hline Teacher & $428(54.7 \%)$ & $233(52.2 \%)$ & 195 (58.0\%) & \\
\hline Staff & $354(45.3 \%)$ & $213(47.8 \%)$ & $141(42.0 \%)$ & \\
\hline Baseline BMI & & & & .34 \\
\hline$<25.0$ & $275(35.2 \%)$ & $156(35.0 \%)$ & $119(35.4 \%)$ & \\
\hline $25.0-29.9$ & $280(35.8 \%)$ & $152(34.1 \%)$ & $128(38.1 \%)$ & \\
\hline$\geq 30.0$ & $227(29.0 \%)$ & $138(30.9 \%)$ & $89(26.5 \%)$ & \\
\hline Trying to lose weight & & & & .71 \\
\hline Yes & $511(65.3 \%)$ & $289(64.8 \%)$ & $222(66.1 \%)$ & \\
\hline No & $271(34.7 \%)$ & $157(35.2 \%)$ & $114(33.9 \%)$ & \\
\hline
\end{tabular}

may result in large population benefit (Rose, 1985). Given the widespread prevalence of overweight and obesity and limited reach of intensive weight loss programs, interventions producing small shifts in weight that can be disseminated broadly have the potential to produce significant population health benefits. Additionally, the difference in weight change between conditions in this study increased over time, in contrast to intensive weight loss programs where the magnitude of difference between conditions tends to lessen over time.

For environmental and policy-focused interventions such as the ones tested in this trial to be successful in the long-term, a commitment is needed to continued, ongoing implementation. Environmental and policy-focused interventions lend themselves well to sustained implementation, as they are usually less time- and/or resource-intensive for participants compared to more intensive weight loss programs, thus promoting intervention adherence and retention, It is for these reasons that such interventions are often recommended due to their potential for sustainability. While the two year follow-up period included in this study exceeds what is typically evaluated in weight loss interventions, it is not long enough to infer long-term intervention impact.

Our findings build upon findings from a recent systematic review of weight gain prevention interventions conducted in workplace and college settings (Gudzune et al., 2013). This review demonstrated

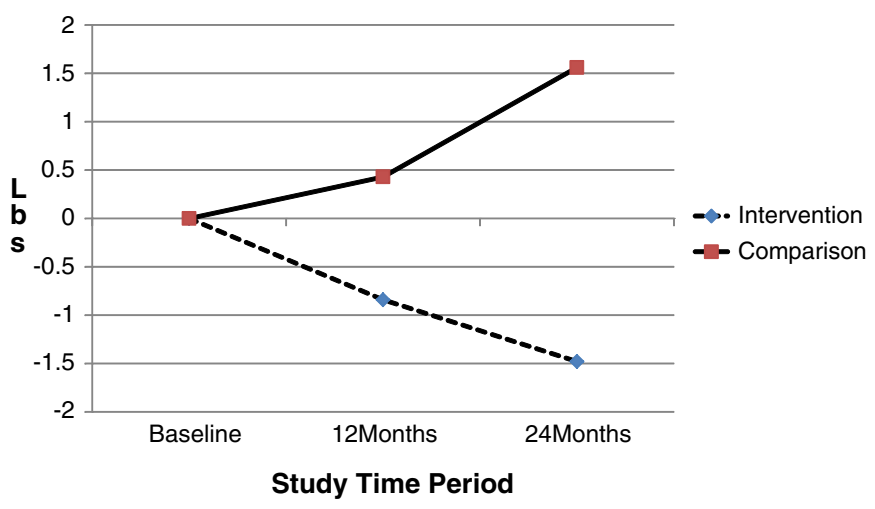

Fig. 2. Estimates of mean weight change by study condition in a multi-level weight gain prevention intervention trial conducted among public school employees in central Massachusetts, 2010-2012. moderate support (prevention of approximately $\geq 0.5 \mathrm{~kg}$ over 1 year) for interventions that combined a variety of strategies at multiple levels including individual counseling and environmental strategies that improve access to healthy food options and physical activity compared to control conditions. We found that an approach that targeted physical and social environments, without individual counseling, achieved similar results in school worksites and can be extended over a two-year period.

Few studies specifically examined weight control interventions for school employees. These studies have assessed individually targeted programs such as weight loss programs and educational strategies (Aldana et al., 2005; Blair et al., 1986). In the most recently published and most well-designed of these studies, Siegel and colleagues observed a similar net change in BMI (.56 units) using an intervention model that involved multiple dietary and physical activity strategies (e.g. healthy snacks at meetings, walking clubs, cooking classes) that were directed by an employee oversight board (Siegel et al., 2010). Our study contributes to this literature by implementing and evaluating an intervention specifically targeting the physical and social worksite environment through multi-level intervention strategies, which are becoming increasingly standard in worksite health promotion approaches (Lemon and Estabrook, in press).

In developing the current intervention, particular attention was paid to integrating the intervention within the organizational facilities, structure, and culture of the school worksite environment. Unlike many other types of worksites, most schools have an existing physical infrastructure in which weight gain prevention programming can be integrated. These include facilities that can support weight control programs for employees and can be targeted to support healthy diets (e.g., cafeterias, faculty and staff lounges, vending machines), weight monitoring (e.g., scales in nurses' offices) and physical activity (e.g., gymnasiums, fitness rooms and equipment, outdoor activity areas, showering facilities) (Burgeson et al., 2003; Grunbaum et al., 2001). The majority of schools also have school nurses on site that can be engaged in health-promoting activities for the entire school population as part of their job responsibilities and are seen as a resource for health promotion by co-workers. The multi-level intervention model in this study utilized school nurses as intervention coordinators and school employee advisory boards to prioritize and tailor intervention strategies to the unique culture of the school. The design and implementation of an intervention model that could be easily integrated within the school worksite social context with modest additional cost (about $\$ 3000$ per school per year) was thus an important driver of the intervention's successful effect on weight gain prevention.

Of the three levels targeted by the intervention, policy change was the most challenging area in which to achieve success, as described in Table 3. There may be inherent barriers to explicit policy change in the public institution setting, where there is intense local scrutiny of public employees and the benefits they receive. School administrators often were willing to make de facto changes, for example, to allow employees to exercise during the school day or to use student facilities, but were not willing or able to make a formal policy. When policy changes were not feasible, some schools made changes to the staff handbook, thus encouraging staff behaviors that support healthy weight without rising to the level of policy.

This study has numerous strengths. Public schools across the U.S. have similar missions, organizational structures and facilities, making the study potentially generalizable nationally. Additional study strengths include the cluster randomized study design, high retention rate, and longer follow-up duration compared to most weight loss intervention studies. Study limitations include the utilization of a convenience sample of employees, which represented $53 \%$ of the total workforce. The study sample was predominantly non-Latino white and educated, which limits the generalizability of the study's findings to other populations that are diverse with respect to race/ethnicity and socioeconomic status. Measures of intervention participation were 
Table 2

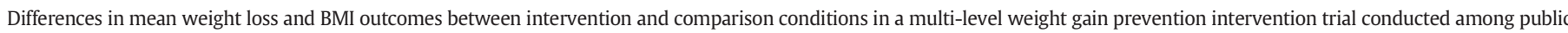
school employees in central Massachusetts, 2010-2012.

\begin{tabular}{|c|c|c|c|c|c|c|c|c|}
\hline \multirow[b]{3}{*}{ Outcome } & \multicolumn{4}{|l|}{12 Month } & \multicolumn{4}{|l|}{24 Month } \\
\hline & \multicolumn{4}{|l|}{$95 \% \mathrm{CI}$} & \multicolumn{4}{|l|}{$95 \% \mathrm{CI}$} \\
\hline & Difference & Lower & Upper & P-value & Difference & Lower & Upper & $P$-value \\
\hline Weight (lbs.) & -1.27 & -3.38 & 0.85 & .24 & -3.03 & -5.85 & -0.22 & .04 \\
\hline BMI $\left(\mathrm{kg} / \mathrm{m}^{2}\right)$ & -0.20 & -0.58 & 0.19 & .32 & -0.48 & -0.96 & 0.00 & .05 \\
\hline
\end{tabular}

collected via self-report and are thus subject to recall and social desirability bias. The two year follow-up period was not long enough to examine if clinically significant differences between conditions can be achieved over time.

\section{Conclusion}

This study observed a modest impact on weight outcomes, demonstrating weight gain prevention can be achieved in a low-intensity, school worksite-based intervention that targets employee physical and social environments. Worksites, where a large percentage of adults spend significant amounts of time, are an important venue for health promotion. As worksite health promotion increases in prominence with provisions provided in the Affordable Care Act, it will be important to develop intervention approaches that are effective within targeted settings. Given the equivocal nature of the weight-related worksite health promotion literature, evidence-based approaches are needed. Although the weight outcomes achieved in this and other worksite interventions do not match the larger impact achieved in highintensity behavioral weight loss programs, as we address the U.S. obesity epidemic, numerous approaches of varying reach and intensity will be required to achieve a population-level impact of stopping and reversing the current weight gain trends. Supportive social and physical environments have an important role from this societal perspective and have particular value as facilitators of weight gain prevention. Continued research is needed to determine intervention strategies that best incorporate social and physical environmental and policy change to maximize the promise of weight loss intervention in worksite settings and to assess the long-term effects of multi-level weight gain prevention strategies.
Conflict of interest statement

The authors declare that there are no conflicts of interests.

\section{Acknowledgments}

We thank Karen Ronayne and Susan Garland for their roles in implementing the intervention and Dante Simone and Nancy O'Mealey for their roles in data collection. We also thank the project coaches, leadership and staff at each of the participating schools. This research was funded by grant number R01 CA132941 from the National Cancer Institute and cooperative agreement number U48 DP001933, awarded to the UMass Worcester Prevention Research Center through the CDC Prevention Research Center program.

\section{References}

Aldana, S.G., Merrill, R.M., Price, K., Hardy, A., Hager, R., 2005. Financial impact of a comprehensive multisite workplace health promotion program. Prev. Med. 40, 131-137.

Blair, S.N., Smith, M., Collingwood, T.R., Reynolds, R., Prentice, M.C., Sterling, C.L., 1986. Health promotion for educators: impact on absenteeism. Prev. Med. 15, 166-175.

Blumenthal, D., 2006. Employer-sponsored health insurance in the United States-origins and implications. N. Engl. J. Med. 355, 82-88.

Burgeson, C.R., Wechsler, H., Brener, N.D., Young, J.C., Spain, C.G., 2003. Physical education and activity: results from the school health policies and programs study 2000. (Special Report) (Excerpt). JOPERD J. Phys. Educ. Recreat. dance 74 (1), 19+.

Cahill, K., Moher, M., Lancaster, T., 2008. Workplace interventions for smoking cessation. Cochrane Database Syst. Rev. CD003440.

Centers for Disease Control and Prevention, 2013. Components of coordinated school health. from http://www.cdc.gov/healthyyouth/cshp/components.htm (Accessed June 17, 2013).

Centers for Disease Control and Prevention, 2012. Workplace health model. from http:// www.cdc.gov/workplacehealthpromotion/model/index.html (Accessed June 17, 2013).

Table 3

Intervention implementation and participation in multi-level intervention condition at 6 public high schools in central Massachusetts, $2010-2012$.

\begin{tabular}{|c|c|c|c|c|c|}
\hline \multirow[t]{2}{*}{ Level of intervention strategies } & \multicolumn{2}{|l|}{ School worksite implementation } & \multicolumn{3}{|l|}{ Employee participation } \\
\hline & & $\begin{array}{l}N=6 \text { schools } \\
N(\%)\end{array}$ & & $\begin{array}{l}12 \text { Month } \\
N=382 \\
\mathrm{~N}(\%)\end{array}$ & $\begin{array}{l}24 \text { Month } \\
N=348 \\
N(\%)\end{array}$ \\
\hline \multirow{9}{*}{$\begin{array}{l}\text { Organizational (physical } \\
\text { environment and policy } \\
\text { interventions) }\end{array}$} & Provided onsite group fitness classes & $6(100 \%)$ & Attended fitness classes & $91(23.8)$ & $74(21.3)$ \\
\hline & Provided school walking route maps & $6(100 \%)$ & Used walking maps & $97(25.4)$ & $75(21.5)$ \\
\hline & Provided access to onsite fitness facilities & $6(100 \%)$ & Used school gym or fitness room & $108(28.3)$ & $85(24.4)$ \\
\hline & Arranged pre-order service for healthy lunch & $2(33.3 \%)$ & Pre-ordered healthy lunch & $35(9.2)$ & $30(8.6)$ \\
\hline & Provided nutrition information at point of selection & $1(16.7 \%)$ & & & \\
\hline & $\begin{array}{l}\text { Reduced number of sugar sweetened beverages in vending } \\
\text { machines }\end{array}$ & $1(16.7 \%)$ & & & \\
\hline & Moved fresh whole fruit to high traffic location in cafeteria & $1(16.7 \%)$ & & & \\
\hline & Promoted formation of walking groups & $6(100 \%)$ & Participated in a walking group & $150(39.3)$ & $100(28.7)$ \\
\hline & Organized physical activity challenges & $6(100 \%)$ & Participated in a physical activity challenge & $217(56.8)$ & $125(35.9)$ \\
\hline \multirow{4}{*}{$\begin{array}{l}\text { Interpersonal } \\
\quad \text { (social environment) }\end{array}$} & Provided or promoted staff games, $5 \mathrm{~K}$ walk/run, other events & $5(83.3 \%)$ & & & \\
\hline & Organized healthy potlucks & $2(33.3 \%)$ & Participated in healthy potluck & $40(10.5)$ & $49(14.1)$ \\
\hline & Provided strength training workshop & $6(100 \%)$ & Attended strength training workshop & $43(11.3)$ & $18(5.2)$ \\
\hline & Provision of healthy eating information display and tasting & $6(100 \%)$ & Attended healthy eating display and tasting & $170(44.5)$ & $156(44.8)$ \\
\hline \multirow[t]{4}{*}{ Individual } & Provided Employee Resource Book & $6(100 \%)$ & Used Employee Resource Book & $242(63.3)$ & $249(71.5)$ \\
\hline & Provided Step Ahead website & $6(100 \%)$ & Visited Step Ahead website & $91(23.8)$ & $112(32.2)$ \\
\hline & Provided Step Ahead newsletter & $6(100 \%)$ & Read Step Ahead newsletter & $228(59.7)$ & $228(65.5)$ \\
\hline & Provided pedometers & $6(100 \%)$ & Used pedometer & $282(73.8)$ & $140(40.2)$ \\
\hline
\end{tabular}


Dunn, C., Whetstone, L.M., Kolasa, K.M., et al., 2013. Delivering a behavior-change weight management program to teachers and state employees in North Carolina. Am. J. Health Promot. 26, 378-383.

Finkelstein, E.A., Trogdon, J.G., Cohen, J., Dietz, W., 2009. Annual medical spending attributable to obesity: payer-and service-specific estimates. Health Aff. 28, w822-w831.

Flegal, K.M., Carroll, M.D., Kit, B.K., Ogden, C.L., 2012. Prevalence of obesity and trends in the distribution of body mass index among US adults, 1999-2010. JAMA 307, 491-497.

Goetzel, R.Z., Ozminkowski, R.J., 2008. The health and cost benefits of work site healthpromotion programs. Annu. Rev. Public Health 29, 303-323.

Grunbaum, J.A., Rutman, S.J., Sathrum, P.R., 2001. Faculty and staff health promotion: results from the School Health Policies and Programs Study 2000. J. Sch. Health 71, 335-339.

Gudzune, K., Hutfless, S., Maruthur, N., Wilson, R., Segal, J., 2013. Strategies to prevent weight gain in workplace and college settings: a systematic review. Prev. Med. 57 268-277.

Hennrikus, D.J., Jeffery, R.W., 1996. Worksite intervention for weight control: a review of the literature. Am. J. Health Promot. 10, 471-498.

Hill, J.O., Wyatt, H.R., Reed, G.W., Peters, J.C., 2003. Obesity and the environment: where do we go from here? Science 299, 853-855.

Janer, G., Sala, M., Kogevinas, M., 2002. Health promotion trials at worksites and risk factors for cancer. Scand. J. Work Environ. Health 28, 141-157.

Kamphuis, C.B., Giskes, K., de Bruijn, G.J., Wendel-Vos, W., Brug, J., van Lenthe, F.J., 2006. Environmental determinants of fruit and vegetable consumption among adults: a systematic review. Br. J. Nutr. 96, 620-635.

Lemon, S.C., Estabrook, B., 2013. Prevention and management of chronic disease through worksite health promotion, In: Reikert, K.A., Ockene, J.K., Pbert, L. (Eds.), Handbook for Health Behavior Change, 4th ed. Springer Publishing Company, New York.

Lemon, S.C., Zapka, J., Li, W., et al., 2010. Step Ahead: a worksite obesity prevention tria among hospital employees. Am. J. Prev. Med. 38, 27-38.

Lewis, C.E., Jacobs Jr., D.R., McCreath, H., et al., 2000. Weight gain continues in the 1990s: 10 -year trends in weight and overweight from the CARDIA study. Coronary Artery Risk Development in Young Adults. Am. J. Epidemiol. 151, 1172-1181.

Mattke, S., Liu, H., Caloyeras, J., et al., 2013. Workplace Wellness Programs Study: Final Report. Rand Corporation, Santa Monica, CA

Ogden, C.L. Carroll, M.D., Kit, B.K., Flegal, K.M., 2012. Prevalence of obesity in the United States, 2009-2010. NCHS data brief, no 82. National Center for Health Statistics, Hyattsville, MD.

Resnicow, K., Davis, M., Smith, M., et al., 1998. Results of the TeachWell worksite wellness program. Am. J. Public Health 88, 250-257.

Rose, G., 1985. Sick individuals and sick populations. Int. J. Epidemiol. 14, 32-38.
Sallis, J., Owen, N., 1999. Physical Activity and Behavioral Medicine. Sage Publications, Thousand Oaks, CA

Sallis, J.F., Owen, N., Fisher, E.B., 2008. Ecological models of health behavior, In: Glanz, K., Rimer, B.K., Viswanath, K. (Eds.), Health Behavior and Health Education. Theory, Research, and Practice, 4th ed. Jossey-Bass, San Francisco, CA.

Schulz, K.F., Altman, D.G., Moher, D., 2010. CONSORT 2010 statement: updated guidelines for reporting parallel group randomized trials. Ann. Intern. Med. 152, 726-732.

Shaikh, A.R, Yaroch, A.L, Nebeling L Yeh, M.C., Resnicow, K, 2008. Psychosocial predictors of fruit and vegetable consumption in adults a review of the literature. Am. J. Prev. Med. 34, 535-543.

Siegel, J.M., Prelip, M.L., Erausquin, J.T., Kim, S.A., 2010. A worksite obesity intervention: results from a group-randomized trial. Am. J. Public Health 100, 327-333.

Task Force on Community Prevention Services, 2009. A recommendation to improve employee weight status through worksite health promotion programs targeting nutrition, physical activity, or both. http://www.thecommunityguide.org/obesity/ RecommendationImproveEmployeeWeightStatusWorksiteHealthPromotion ProgramsTargetingNutritionPhysicalActivityorBoth.pdf (Accessed June 17, 2013).

The Robert Wood Johnson Foundation, 2012. Health Affairs: Health policy brief. Workplace wellness programs. http://www.healthaffairs.org/healthpolicybriefs/brief.php? brief_id=69 (Accessed June 17, 2013).

Thorndike, A.N., 2011. Workplace interventions to reduce obesity and cardiometabolic risk. Curr Cardiovasc Risk Rep. 5, 79-85.

Trost, S.G., Owen, N., Bauman, A.E., Sallis, J.F., Brown, W., 2002. Correlates of adults' participation in physical activity: review and update. Med. Sci. Sports Exerc. 34, 1996-2001.

U.S. Department of Health and Human Services, 2012. Healthy people 2020 - Improving the health of Americans. http://www.healthypeople.gov/2020/default.aspx (Accessed June 17, 2013).

U.S. Department of Labor \& Division of Labor Force Statistics, 2012. Employment status of the civilian population by sex and race. http://www.bls.gov/news.release/empsit.t01. htm (Accessed June 17, 2013).

U.S. Government, 2010. Public Law 111-296. Healthy Hunger-Free Kids Act of 2010. http://www.gpo.gov/fdsys/pkg/PLAW-111publ296/pdf/PLAW-111publ296.pdf (Accessed June 17, 2013).

U.S. Government Printing Office, 2010. Patient protection and affordable care act (No. Public Law No. 111-148, page 124 Stat 119). US Government Printing Office, Washington, DC March.

Verweij, L.M., Coffeng, J., van Mechelen, W., Proper, K.I., 2010. Meta-analyses of workplace physical activity and dietary behaviour interventions on weight outcomes. Obes. Rev. 12, 406-429.

Zapka, J., Lemon, S.C., Estabrook, B., Jolicoeur, D., 2007. Keeping a Step Ahead - formative phase of a workplace intervention trial to prevent obesity. Obesity $15,27 \mathrm{~S}-36 \mathrm{~S}$. 